\title{
Landesparlamente im unitarischen Bundesstaat: „Machtlosigkeit“ und „unheilige Allianz“
}

\author{
Werner Reutter ${ }^{1, *}$ \\ 1 Institut für Sozialwissenschaften, Humboldt-Universität zu Berlin, Germany \\ * werner.reutter@rz.hu-berlin.de
}

\section{Zusammenfassung}

Gemeinhin gelten Landesparlamente im bundesdeutschen Föderalismus als quantité négligeable. Ihre Macht scheint, so die überwiegende Auffassung, eng beschränkt, weil im unitarischen Bundesstaat deutscher Prägung alle wichtigen Entscheidungen auf zentralstaatlicher Ebene getroffen werden und die Volksvertretungen in den Bundesländern nicht wirkmächtig an der Staatsleitung beteiligt sind. Die empirische Analyse zeigt allerdings, dass von einem kontinuierlichen Macht- und Einflussverlust der Landesparlamente nicht die Rede sein kann. Weder hat sich der „legislative Gestaltungsraum“ der Bundesländer stetig verkleinert, noch kann davon gesprochen werden, dass Landesparlamente auf Gesetzgebung ohne Einfluss bleiben.

\section{Schlüsselwörter}

Föderalismus, Parlamentarismus, Verfassungskonflikt, Gesetzgebung, Verfassungspolitik

\section{Land parliaments and unitarian federal state: between „powerlessness“ and „unsacred alliance“}

\begin{abstract}
In general, in German federalism state parliaments are supposed to be hardly more than a quantité négligeable. In this perspective, the power of state parliaments is severely limited in two ways: On the one hand, in the German unitary federal state all essential decisions are being made at the national level, on the other hand state parliaments cannot effectively participate in policy making at the subnational level. Yet, from an empirical point of view these assumptions turn out to be false. State parliaments did neither continuously lose power over time nor has their capacity to legislate steadily diminished. Theoretically, the findings presented in this paper question current approaches trying to explain status and influence of German state parliaments.
\end{abstract}

\section{Keywords}

Federalism, parliamentarism, constitutional conflict, legislation, constitutional politics

The author has declared that no competing interests exist. 
In der politikwissenschaftlichen Diskussion um Rolle und Bedeutung der Landesparlamente in der Bundesrepublik Deutschland wird überwiegend die Auffassung vertreten, dass die Vertretungskörperschaften in den Bundesländern einen kontinuierlichen Macht- und Einflussverlust erfahren hätten und nicht mehr wirkmächtig an der Staatsleitung in den Ländern beteiligt seien. Gerne wird in diesem Zusammenhang das Wort vom "Niedergang“ benutzt. Die These eines "Niedergangs der Parlamente" hat in der Bundesrepublik Deutschland immer wieder Anklang gefunden. Auch aktuell leben wir in einer "postparlamentarischen“ Demokratie (Crouch 2008) mit "entmachteten“ Parlamenten (Grimm 200I), die für nicht wenige $\mathrm{zu}$ „Abnickvereinen“" (Müller 20II; Oberreuter 20I3, 2I-22), zu „staatsnotarielle[n] Ratifikationsämter[n]“ (Kilper/Lhotta 1996, 202) oder zu "Ratifikationsinstanzen“ (Papier 2013, 83) verkommen sind. Für Hans Herbert von Arnim (2002, 162) war der Parlamentarismus in den Bundesländern sogar schon um die Jahrtausendwende "abgeschafft". Im kooperativen Föderalismus deutscher Prägung kann es offenbar nur heißen: Landesparlamente „don't matter!“ (Eicher I988, 76-IOI; Klatt 1982; Linck 2004). Als verantwortlich für diese Entwicklungen gelten - neben der europäischen Integration, die im Weiteren unberücksichtigt bleibt - zwei sich gegenseitig verstärkende Faktoren: die Politikverflechtung im kooperativen Föderalismus mit ihren Kooperationszwängen und Unitarisierungstendenzen einerseits sowie die Funktionsmechanismen parlamentarischer Regierungssysteme andererseits. In dieser Perspektive bilden Föderalismus und Parlamentarismus eine „unheilige Allianz", die Macht und Einfluss von Volksvertretungen in den Ländern erodieren lassen (Lehmbruch 2000; Scharpf et al. 1976; Kropp 20I0). Aus diesen Gründen hat Uwe Thaysen schon 2005 konstatiert, dass sich der Landesparlamentarismus in einem "Überlebenskampf" befinde (Thaysen, 2005, 32; Reutter 2008, 19-26).

In diesem Beitrag wird der Erklärungsgehalt dieser Positionen diskutiert und überprüft. Insbesondere wird der Annahme nachgegangen, dass der kooperative Föderalismus deutscher Prägung einen landesparlamentarischen Machtverlust bedingt habe, weil er den legislativen Gestaltungsraum der Bundesländer sukzessive verkleinert und die Exekutive gegenüber der Legislative privilegiert habe. Empirisch überprüft wird dies anhand der Gesetzgebungsfunktion, die auch in den skizzierten kritischen Positionen meist herangezogen wird, um den Landesparlamenten einen Macht- und Einflussverlust zu attestieren. ${ }^{1}$ Dabei stehen zwei Argumente im Zentrum der Analyse: einerseits die Behauptung, der legislative Gestaltungsraum der Bundesländer sei kontinuierlich eingeschränkt worden, sowie andererseits die Vermutung, Landesparlamente könnten

I Die Niedergangsthese ließe sich auch anhand anderer Parlamentsfunktionen prüfen; aus Platzgründen und weil sich die einschlägige Literatur vorwiegend, bisweilen sogar ausschließlich auf die Gesetzgebung bezieht, beschränke ich mich im Weiteren auf diesen Aufgabenbereich. sich nicht mehr wirkmächtig an der Staatsleitung und am Policy Making beteiligen. Dafür werden zuerst die "Niedergangsthese" und ihre Annahmen dargelegt. Darauf aufbauend wird herausgearbeitet, ob die Entwicklung des unitarischen Bundesstaates von einer „Entparlamentarisierung" subnationaler Vertretungskörperschaften begleitet war. Sodann soll auf Grundlage von Studien aus der vergleichenden Parlamentarismusforschung der Einfluss der Landesparlamente auf Policy Making empirisch erschlossen werden. Abschließend werden die präsentierten Befunde zusammengefasst und theoretisch eingeordnet. ${ }^{2}$ Wohlgemerkt geht es im Weiteren nicht darum, aus Theorien abgeleitete Hypothesen oder kausale Beziehungen über den Zusammenhang von Bundesstaat und Landesparlamentarismus zu "testen“. Vielmehr sollen auf Grundlage von erhobenen Daten in der einschlägigen Forschung verbreitete Argumente hinterfragt und kritisch diskutiert werden.

\section{Niedergang des Landesparlamentarismus: aktuelle Diagnosen}

Parlamentarismuskritik scheint so alt wie der Parlamentarismus selbst. Zum ersten Mal wurde ein „Niedergang des Parlamentarismus" vor fast Ioo Jahren diagnostiziert (Bryce I92I, 335-357; Schmitt I923/I988; Habermas 1993; Marschall 2005, 252-28I; Beyme I999, I62-I77). Auch Landesparlamenten werden seit langer Zeit ausgreifende Funktionsdefizite und ein kontinuierlicher Machtverlust attestiert (Carstensen/Schüttemeyer 2015, 284-285; Leber 2014, 7I-76; Reutter 2008, 22-26 und 339-35I; Hennis 1956/1968; Mende 2010; Friedrich 1975; Eicher 1988; Linck 2004; Thaysen 2005; Papier 20I3). Drei Argumente werden in diesem Zusammenhang vorgebracht: Erstens wird nicht selten die Vergangenheit idealisiert und eine "goldene Ära des Parlamentarismus" beschworen. So misst Hans-Jörg Eisele die Performanz des Landtages Baden-Württemberg an einem "kraftvollen Landesparlamentarismus", der früher einmal existiert habe und dessen Rückkehr wünschens- und erstrebenswert sei. Wie dieser „kraftvolle Landesparlamentarismus" in der Vergangenheit ausgesehen hat oder wie er in Zukunft aussehen soll, wird nicht erläutert. Gleichwohl dient der „kraftvolle Landesparlamentarismus“ als Maßstab,

2 Die Auswahl der für die Analyse herangezogenen Landesparlamente orientiert sich an zwei Kriterien: an der Zugänglichkeit der notwendigen Daten sowie daran, dass die Bundesländer möglichst „repräsentativ“ abgebildet werden. Es sollten mithin Landesparlamente aus „alten“ und „neuen“ Bundesländern, aus Süd-wie aus Norddeutschland sowie mit unterschiedlichen parteipolitischen Traditionen aufgenommen werden. Nicht in die Analyse aufgenommen wurden die Stadtstaaten Hamburg, Berlin und Bremen. 
um der Parlamentswirklichkeit der Gegenwart Defizite insbesondere im Bereich der Gesetzgebung zu attestieren oder allgemein einen "verfassungsrechtlichen und politischen Verfallsprozess" zu beklagen (Papier 20I3, 87). Verbreitet ist, zweitens, die Annahme, dass Demokratie und Parlamentarismus auseinanderfallen. So habe der Landtag Baden-Württemberg nach Hans-Jörg Eisele nur noch eine „formale Existenzberechtigung“" (Eisele 2006, 377). Landesparlamente beschließen danach zwar weiterhin Gesetze, kontrollieren die Regierung oder debattieren über öffentliche Angelegenheiten. Allerdings entfalten diese Aktivitäten, so Kritiker, keine substanzielle demokratische Wirkung (Linck 2004; Hennis 1956/1968). Sie sind, wie Hans-Jürgen Papier (2OI3, 83) feststellt, „Form“ ohne "materielle[s] Substrat". Landesparlamente sind in dieser Perspektive nicht mehr effektiv an der Staatsleitung beteiligt. Hinzu kommt, drittens, dass, so Carstensen und Schüttemeyer $(2015,286)$, in den Analysen zwar ein kontinuierlicher Kompetenz- und Machtverlust der Landesparlamente konstatiert wird, dieser sich aber in unterschiedlichen Funktionsbereichen zu manifestieren scheint. Während manche vor allem die Gesetzgebung und damit die Fähigkeit zur politischen Gestaltung beeinträchtigt sehen (Eisele 2006; Papier 2013; Mende 20Io), meinen andere vor allem Defizite bei der Kontroll- oder der Kommunikationsfunktion ausgemacht zu haben (Oberreuter 2012; ders. 2013). Doch unbeschadet dieser Differenzen in der Wirkung stimmen die Autoren in ihrer Erklärung dieser Funktionsdefizite überein. Erwähnt werden hier vor allem: kooperativer Föderalismus und Europa (Thaysen 2002, ders. 2005; Marschall 2005, I99-20I; Reutter 2008).

Aus dem Überblick lassen sich zwei grundlegende Annahmen zur Macht und zum Einfluss von Landesparlamenten ableiten: Zum einen unterstellt die Niedergangsthese, dass Landesparlamente einen kontinuierlichen Macht- und Einflussverlust erfahren haben. Belegt wird diese Annahme damit, dass die Gesetzgebungskompetenzen des Bundes zugenommen hätten (Eicher I988; Thaysen 2002). Das ist allerdings lediglich eine indirekte Beweisführung. Denn es besteht durchaus die Möglichkeit, dass auf beiden Ebenen des Bundesstaates neue Politikbereiche entstehen, die eine legislative Gestaltung erfordern. Anders gesagt: der legislative Gestaltungsraum von Bund und Ländern kann gleichzeitig erweitert - oder auch verkleinert - werden. Der Machtund Einflussverlust von Landesparlamenten muss folglich auf Ebene der Bundesländer erschlossen werden; er lässt sich nicht ableiten aus der Erweiterung der legislativen Kompetenzen des Bundes im Rahmen der konkurrierenden und ausschließlichen Gesetzgebung. Im Weiteren werden daher Anzahl und Qualität von in den Bundesländern verabschiedeten Gesetzen als Maßstab genommen, um einen Macht- und Einflussverlust festzustellen. Konkret heißt dies: dass in den Bundeslän- dern im historischen Verlauf weniger und zunehmend unwichtige Gesetze verabschiedet werden und dass die Gesetzgebungstätigkeit in den Bundesländern aufgrund der homogenen Rahmenbedingungen ein kongruentes Profil aufweisen sollte. Zusammenfassend lässt sich sagen: Sollte die Annahme zutreffen, dass Landesparlamente stetig an Macht und Einfluss verloren haben, sollte der Raum, den diese Legislativorgane gestalten können, kleiner geworden sein. Zum anderen sollte der Einfluss, den Landesparlamente auf die Gestaltung dieses Raumes ausüben können, abgenommen haben. Landesparlamente können also an den den Bundesländern zustehenden legislativen Kompetenzen nicht oder nicht mehr effektiv mitwirken. Dahinter steht die Vermutung, dass mit Unitarisierungstendenzen und Politikverflechtung Landesparlamente zu reinen Policy Takern geworden sind, die nur noch das nachvollziehen, was die politische Exekutive in anderen Zusammenhängen beschlossen hat. Landesparlamente sind also nur noch formal an der Formulierung und Verabschiedung von Gesetzen beteiligt; die substanziellen Entscheidungen werden dagegen außerparlamentarisch getroffen.

\section{Der Einfluss von Landesparlamenten im Bundesstaat: methodische Überlegungen, "legislativer Gestaltungsraum“ und „Performanz"}

Die Antwort auf die Frage, wie viel Einfluss Parlamente an der Staatsleitung oder am Policy Making haben, ist voraussetzungsvoll. Sie umfasst: methodische Überlegungen, die Bestimmung eines legislativen Raumes („capacity“) sowie die empirische Analyse des Einflusses („performance“).

\subsection{Methodische Überlegungen zur Analyse des Ein- flusses von Parlamenten}

In seiner bahnbrechenden Studie Comparative Legislatures untersucht Michael L. Mezey (1979) den Einfluss von Parlamenten auf Policy Making. In diesem Kontext stellt Mezey seine die Forschung befruchtende Frage, ob und inwieweit ein Parlament Policy Making beeinflussen kann, also wirkmächtig an der Gesetzgebung beteiligt ist. Das ist die sogenannte "Mezey question“ (Arter 2006b). Ein Parlament, so Mezey, hat Einfluss, wenn es Gesetze mit einem Veto verhindern kann. „Legislatures will be salient in the policy-making process to the extent that their presence and prerogatives act as a constraint on the executive. [...] The most telling constraint that the legislature can place on the policy-making process is the veto" (Mezey 1979, 25). Unter Hinzuziehung eines zweiten Kriteriums - der öffentlichen Unterstützung unterscheidet Mezey fünf Typen von Legislatures: die „Vulnerable Legislatures“ (Philippinen, Uruguay etc.), 
die "Marginal Legislatures“ (Thailand, Pakistan, Südvietnam [vor 1975] etc.), die "Active Legislatures" (US Kongress, Costa Rica), die „Reactive Legislatures“ (UK, Kanada, BRD etc.) sowie die "Minimal Legislatures“ (Sowjetunion, Polen etc.). Die Zuordnung der Länder ergibt sich aus den beiden genannten Kriterien: der „Policy-Making Power" und der öffentlichen Unterstützung (Mezey 1979, 36-37). In der vergleichenden Parlamentarismusforschung standen "reactive" und "active legislatures" im Zentrum. Auch für die vorliegende Untersuchung ist allein der Typus der "reactive legislature“ - dazu zählt z.B. der Deutsche Bundestag und das British House of Commons - einschlägig; er findet sich in Demokratien mit parlamentarischen Regierungssystemen (Mezey 1979, 39) oder eben in den von Mezey nicht berücksichtigten deutschen Bundesländern (Freitag/Vatter 2008). Ein solches Parlament erfährt grundsätzlich öffentliche Akzeptanz und übt einen "mittleren“ Einfluss auf Policy Making aus. Es verfügt zwar nicht - so Mezey bezogen auf das House of Commons - über "the effective power to say no to the government". Aber es kann "certain parameters" setzen, "within which the government must act and thereby discourages the government from introducing legislation that will cause a row in Parliament" (Mezey 1979, 39). Doch ändert das nichts daran, dass in "reactive legislatures" die Exekutive den Gesetzgebungsprozess in seinen drei Stadien dominiert: der Formulierungs-, der Beratungs- und der Kontrollphase.

Dieser Ansatz hat die vergleichende Parlamentarismusforschung nachhaltig befruchtet, aber auch Kritik erfahren (Damgaard/Jensen 2006, Russell et al. 2015; Norton 1990, 5), wobei insbesondere angeführt wird, dass präzise Maßstäbe und Indikatoren fehlen, um die Performanz und den Einfluss von Parlamenten auf die Gesetzgebung zu bestimmen (Arter 2006a, 2006b). Auch die Abgrenzung der Kategorien und die Zuordnung der Fälle werden in Frage gestellt. So überzeugt kaum - wie Mezey unterstellt -, dass „vulnerable legislatures“ über ebenso große „Policy Making Power“ verfügen wie „active legislatures" und sie sich lediglich im Grad der öffentlichen Unterstützung voneinander unterscheiden. Nach Arter könne es daher nicht darum gehen herauszufinden, "how much policy-power" ein Parlament habe, sondern darum, „wie“ Einfluss ausgeübt werde. Mit einer „anatomy of legislative influence“ soll, nach Arter, herausgearbeitet werden, „how do legislators, both individually and collectively, work to perform their legislative roles in the three phases of the legislative process $[\ldots]^{\prime \prime \prime}($ Arter 2006a, 463). Auch die vorliegende Untersuchung zielt auf die Frage, wie Landesparlamente in den unterschiedlichen Stadien von Gesetzgebungsverfahren Einfluss ausüben. Im Anschluss an Arter (2006b) werden dabei zwei analytische Ebenen unterschieden: die "capacity" einerseits sowie die faktische Performanz andererseits. Erstere bezieht sich auf den „legislativen
Gestaltungsraum“, den Landesparlamente ausfüllen können, während letztere darauf abhebt, wie Parlamente Einfluss ausüben.

\section{2 „Capacity“: der legislative Gestaltungsraum der Länder}

Das Grundgesetz kann die Rolle von Landesparlamenten nicht abschließend bestimmen, dies obliegt dem Landesverfassungsgeber. Die Bundesverfassung legt jedoch den "legislativen Gestaltungsraum“ oder in den Worten von David Arter: die "capacity“ von Landesparlamenten fest. Es gibt dabei keine „ideale“ Aufteilung legislativer Kompetenzen zwischen staatlichen Ebenen, die alle normativen Ansprüche, die sich aus dem Demokratiegebot in Mehrebenensystemen ergeben, in gleicher Weise erfüllen kann. Aus der Anzahl und der Qualität der in den Ländern verabschiedeten Gesetze lässt sich gleichwohl ableiten, ob der behauptete „Niedergang des Landesparlamentarismus" in der Verfassungswirklichkeit stattgefunden hat, ob also der „legislative Gestaltungsraum" kleiner geworden ist. Unterstellt wird dabei, dass die Chance von Landesparlamenten, auf Policy Making einzuwirken, mit der Anzahl und der Qualität der zu verabschiedenden Gesetze variiert. Dahinter steht die in der deutschen Föderalismusforschung dominierende Vermutung, dass die bundesstaatliche Kompetenzverteilung sich auf die Macht von Landesparlamenten auswirkt. Die Macht von Landesparlamenten oder die "capacity“ bestimmt sich mithin über die Strukturen der vertikalen Gewaltenteilung im deutschen Bundesstaat. Empirisch überprüfen lässt sich dies in dreierlei Hinsicht: anhand des legislativen Outputs, der in den Bundesländern ein ähnliches Profil aufweisen sollte, anhand der abnehmenden Bedeutung von "wichtigen" Gesetzen sowie anhand der Vermutung, dass Unitarisierungstendenzen zu weniger und Reföderalisierungsmaßnahmen zu mehr Landesgesetzen führen sollten. In der Analyse finden diese Annahmen nur in einer Hinsicht Bestätigung.

Erstens sind beträchtliche Niveauunterschiede festzustellen (Tabelle I), was die Vermutung, der legislative Gestaltungsraum von Landesparlamenten ergebe sich allein aus dem Grundgesetz, zumindest ergänzungsbedürftig erscheinen lässt. Während in Schleswig-Holstein gerade einmal knapp 88 Gesetze pro Wahlperiode verabschiedet wurden, waren es in Bayern rund I27, in Brandenburg, einem neuen Bundesland, sogar I54. Wenn in Betracht gezogen wird, dass alle Bundesländer in denselben föderalen Strukturen eingebunden sind und dem Homogenitätsgebot des Art. 28 Abs. I GG unterliegen, überrascht dieser Befund durchaus. Ebenso stellt sich die Frage, wieso der Bayerische Landtag bis 2013 über 2000 Gesetze verabschiedet hat, während sein Pendant in Schleswig-Holstein bis 2016 rund 600 Gesetze weniger angenommen hat. 
Tabelle 1: Anzahl der verabschiedeten Gesetze in ausgewählten Landesparlamenten

\begin{tabular}{|c|c|c|c|c|c|}
\hline & Zeitraum & Anzahl WP & Anzahl Gesetze & Gesetze pro WP & Gesetze pro Jahr \\
\hline BW & $1953-2016$ & 15 & 1.498 & 106,0 & 25,2 \\
\hline BAY & $1946-2013$ & 16 & 2.036 & 127,3 & 30,4 \\
\hline BB & $1990-2014$ & 5 & 770 & 154,0 & 33,5 \\
\hline HES & $1946-2014$ & 18 & 1.927 & 107,1 & 28,3 \\
\hline MV & $1990-2016$ & 6 & 756 & 126,0 & 29,1 \\
\hline $\mathrm{NI}$ & $1951-2013$ & 16 & 1.778 & 111,1 & 28,7 \\
\hline NRW & $1947-2017$ & 16 & 2.056 & 129,8 & 29,7 \\
\hline $\mathrm{RP}$ & 1947-2011 & 15 & 1.695 & 113,0 & 26,1 \\
\hline SLD & $1970-2009$ & 8 & 774 & 96,8 & 19,8 \\
\hline SN & $1990-2014$ & 5 & 684 & 136,8 & 28,5 \\
\hline SH & 1947-2017 & 18 & 1.405 & 87,8 & 22,7 \\
\hline TH & $1990-2014$ & 5 & 739 & 147,8 & 30,8 \\
\hline Gesamt & & 141 & 16.139 & 114,5 & 26,9 \\
\hline
\end{tabular}

Quelle: Reutter 2013, 67; Homepages der Landesparlamente; eigene Ergänzungen und Berechnungen.

Zweitens, kann die absolute Anzahl von verabschiedeten Gesetzen nur einen ersten Anhaltspunkt dafür liefern, ob und inwieweit sich Strukturen und Funktionsweisen des Bundesstaates auf Macht und Einfluss von Landesparlamenten auswirken können. Ebenso wichtig sind "qualitative“ Dimensionen der verabschiedeten Gesetze (Arter 2006b, 25I). Denn häufig wird angenommen, dass Landesparlamente „Gesetze zweiter Klasse“, also Ausführungs- oder Anpassungsgesetze verabschieden. Danach sollte in den Bundesländern keine „major public policy“ (Loewenberg/Patterson 1979, I97) existieren, die Landesparlamente durch „Schlüsselentscheidungen“ gestalten könnten. Allerdings lassen die wenigen Studien, die sich mit solchen Aspekten beschäftigen, eine solche Schlussfolgerung kaum zu. So ist die Anzahl der „neuen Gesetze“ im Landtag NRW seit 1995 gestiegen (Andersen/ Bovermann 2OI2, 4I9f.). Im Bayerischen Landtag lag der Anteil der „bedeutenden Neuschöpfungen“, die Gremmer über die Länge der Gesetze definiert, bis 1990 bei rund 9 Prozent aller verabschiedeten Gesetze (Gremmer I990, Io8ff.), was deutlich über dem Niveau des Bundestages lag, der nach Klaus von Beyme $(1997,63)$ in I2 Wahlperioden gerade einmal I50 „Schlüsselentscheidungen“ getroffen hat $(=2,5$ Prozent aller verabschiedeten Gesetze). Hinzu kommt, dass auch in einem zentralen Politikfeld der Bundesländer keine abnehmende Tendenz zu verzeichnen ist: in der Verfassungspolitik. Im Gegenteil, wie Abbildung I verdeutlicht, schwankt die Anzahl der pro Jahr und Bundesland verabschiedeten verfassungsändernden Gesetze zwar im Zeitverlauf, doch zeigt sich in diesem Politikfeld eine insgesamt steigende Tendenz.
Während bis 1965 durchschnittlich gerade einmal o,2 Gesetze pro Parlamentsjahr verabschiedet wurden, waren es in den letzten beiden Jahrzehnten doppelt so viele, nämlich 0,4 .

Allerdings ist $\mathrm{zu}$ beachten, dass die Qualifizierung von Gesetzesvorhaben als „wichtig“ oder als „major public policy“ ein „element of informed judgement" beinhaltet (Arter 2006b, 25I). Sie gründet mithin auf sub-

Abbildung 1: Verfassungsändernde Gesetze in den Bundesländern pro Jahr und Parlament (1946 bis 2016)a)

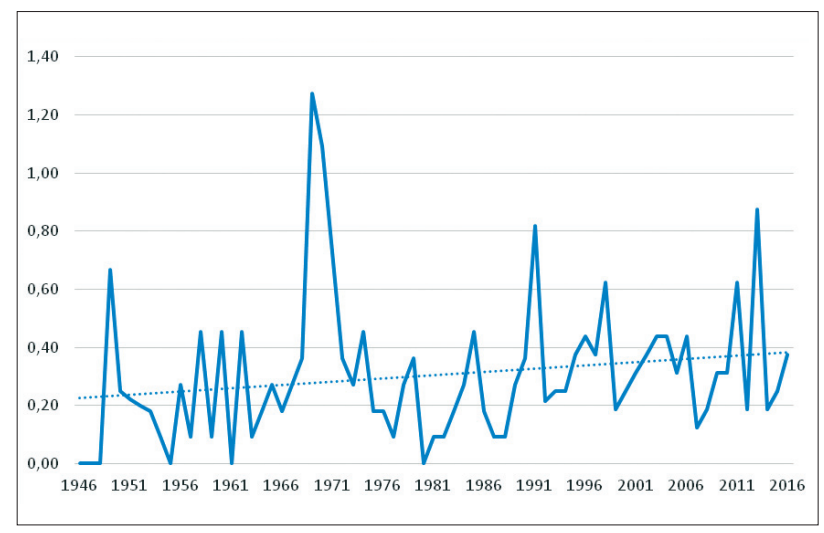

a) Berechnet wurden die Werte, indem die Anzahl der jährlich verabschiedeten verfassungsändernden Gesetze durch die Anzahl der Bundesländer dividiert wurde, die in dem entsprechenden Jahr bereits über eine Verfassung verfügten.

Quelle: eigene Zusammenstellung; Pestalozza 2014a, Parlamentsdokumentationen der Landesparlamente; eigene Ergänzungen und Berechnungen. 
jektiv gefärbte Einschätzungen darüber, was wichtige von unwichtigen Gesetzen trennt (Arter 2006b; Beyme 1997, 65; Reutter 2016b, 97-IOI). Trotzdem gilt: Weder die vorliegenden Studien noch die dargelegten Befunde zur Verfassungspolitik lassen die Schlussfolgerung zu, dass Landesparlamente nicht, nicht mehr oder immer weniger über "major public policies" entscheiden.

Drittens ist festzuhalten, dass die Anzahl der Gesetze keineswegs kontinuierlich zurückgegangen ist. Vielmehr folgt die legislative Performanz in den Bundesländern einem zyklischen Verlauf (Abbildung 2), wobei zu berücksichtigen ist, dass die Io. WP im Hessischen und die 15. im Nordrhein-Westfälischen Landtag verkürzt waren. Doch unbeschadet dieser Ausnahmen zeigt Abbildung 2, dass zumindest in dieser Hinsicht von einem kontinuierlichen Macht- und Einflussverlust der Landesparlamente nicht die Rede sein kann. Jedenfalls manifestierte er sich nicht in einer sinkenden Anzahl verabschiedeter Gesetze pro Wahlperiode und zwar unabhängig davon, wie die legislativen Kompetenzen zwischen Bund und Ländern verteilt waren.

Viertens fällt auf, dass einer Erweiterung der Gesetzgebungskompetenzen des Bundes kein legislativer $\mathrm{Ab}$ schwung auf Landesebene folgte. So sind nach der ersten großen Föderalismusreform Ende der 196oer/Anfang der I970er Jahre, in der die Gesetzgebungskompetenzen des Bundes signifikant erweitert wurden, in den Landesparlamenten die legislativen Aktivitäten keineswegs gesunken. Zumindest wurden in Baden-Württemberg in der 5. und 6. WP (1968/72 und 1972/76) insgesamt I46 bzw. 126 Gesetze verabschiedet und damit deutlich mehr als im Durchschnitt aller Wahlperioden (Io6 Gesetze). Nicht anders war dies in Bayern. Hier wurden in der 6. und 7. WP (1972/75 und 1975/79) I47 bzw. 157 Gesetze vom Landtag angenommen, was ebenfalls weit über dem Durschnitt von 127 Gesetzen pro WP in diesem Bundesland lag. Besonders deutlich hat sich dies bei verfassungsändernden Gesetzen niedergeschlagen: So wurden in diesem Politik-

Abbildung 2: Anzahl der verabschiedeten Gesetze in ausgewählten Bundesländern (seit 1946)

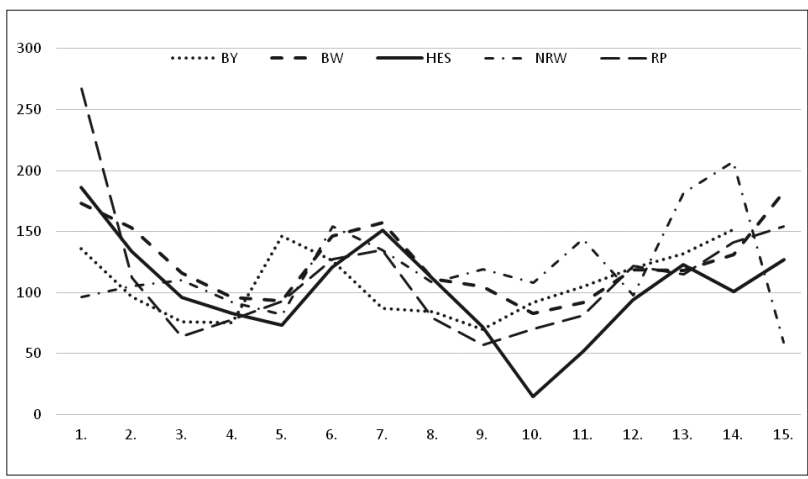

Quelle: Reutter 2013, 67; eigene Ergänzungen; Parlamentsdokumentationen der Landesparlamente. feld bis 1965 insgesamt 39 verfassungsändernde Gesetze verabschiedet, was pro Jahr, in denen eine Verfassungsänderung möglich war (=I88 Jahre), zu einem Wertvon o, 2 führt. In den zehn Jahren danach, also zwischen 1966 und 1976, stieg die Frequenz um das Doppelte, nämlich auf o,5 (57 verfassungsändernde Gesetze; IIo Parlamentsjahre).

Erwartbare Effekte scheinen sich jedoch nach der Föderalismusreform I 2006 eingestellt zu haben, bei der insgesamt I8 Gesetzgebungsmaterien an die Länder übertragen wurden (Leber 20I4; Carstensen/Schüttemeyer 2015, 287-293; Dose/Reus 2016). Zumindest hat der Landtag von Baden-Württemberg in der I4. WP (2006/II) I52 Gesetze angenommen, das war um fast die Hälfte mehr als in der I3. WP (200I/06). Auch wenn Carstensen und Schüttemeyer (2013, 293) davon ausgehen, dass Landesparlamente auch nach der Reform „zentrale Belange [...] ihrer Bürger [...] nach wie vor nicht gestalten können“, bleibt immerhin der Frage, wieso Unitarisierung und Reföderalisierung von Politikfeldern zu ähnlichen Effekten auf Landesebene geführt haben, nämlich zu einem Anstieg verabschiedeter Gesetze. Gleichzeitig zeigte die Analyse, dass von einem kontinuierlichen Machtverlust und einem sich stetig verkleinernden legislativen Gestaltungsraum auf Ebene der Bundesländer nicht die Rede sein kann. Die von vielen geteilte Annahme, dass ein Kompetenzgewinn bzw. -verlust auf Bundesebene eine Einschränkung bzw. Erweiterung der legislativen "capacity“ der Länder nach sich zieht, hält einer empirischen Überprüfung nicht stand.

\section{3. „Performance“: zum Einfluss von Landesparlamenten}

Wie erwähnt, wird meist unterstellt, dass Bundesstaat und Landesparlamentarismus eine "unheilige Allianz" eingegangen sind. In dieser Perspektive geht der kooperative Föderalismus mit Unitarisierungstendenzen einher, weil der Bund legislative Gestaltungskompetenzen erfolgreich für sich reklamiert oder weil die Länder mittels Selbstkoordination ihre Handlungsspielräume freiwillig einschränken. In jedem Fall werde der Einfluss der Landesparlamente auf Policy Making minimiert und derjenige der Exekutive maximiert. Nun sind die $\mathrm{Zu}$ sammenhänge zwischen Föderalismus und Landesparlamentarismus, wie gezeigt, keineswegs so eindeutig wie vielfach unterstellt. Dennoch bleibt zu klären, ob Landesparlamente an der Staatsleitung effektiv mitwirken können. Wie erwähnt, kann es dabei nicht darum gehen, den Einfluss von Landesparlamenten auf Gesetzgebung zu quantifizieren oder Entscheidungen der Exekutiven monokausal auf Entscheidungen der Landesparlamente zurückzuführen. Es geht hier lediglich darum herauszuarbeiten, wie Landesparlamente Einfluss ausüben können und nicht darum, wie viel Einfluss sie ausüben. Dies wird in drei Schritten untersucht: (a) Zuerst wird der In- 
Tabelle 2: Gesetzentwürfe in ausgewählten Landesparlamenten

\begin{tabular}{|c|c|c|c|c|c|c|c|}
\hline \multirow[b]{3}{*}{ BW } & \multirow{3}{*}{$\begin{array}{c}\text { Zeitraum } \\
1952-2016\end{array}$} & \multirow{3}{*}{$\frac{\text { Anzahl WP }}{15}$} & \multicolumn{5}{|c|}{ Gesetzentwürfe ... } \\
\hline & & & \multicolumn{2}{|c|}{... aus dem Landtaga) } & \multicolumn{2}{|c|}{... von Landesregierung } & \multirow{2}{*}{$\frac{\text { Gesamt }}{2.208}$} \\
\hline & & & 786 & 35,6 & 1.422 & 64,4 & \\
\hline BAY & $1946-2013$ & 15 & 1.571 & 51,2 & 1.495 & 48,8 & 3.066 \\
\hline BB & $1990-2014$ & 5 & 240 & 29,0 & 587 & 71,0 & 827 \\
\hline HES & $1995-2008$ & 3 & 219 & 41,1 & 314 & 58,9 & 533 \\
\hline $\mathrm{NI}$ & $1990-2013$ & 5 & 403 & 44,3 & 506 & 55,7 & 909 \\
\hline NRW & $1995-2017$ & 5 & 262 & 29,9 & 638 & 70,1 & 900 \\
\hline $\mathrm{RP}$ & $1947-2011$ & 15 & 698 & 32,6 & 1.445 & 67,4 & 2.143 \\
\hline SLD & $1970-2009$ & 8 & 307 & 32,6 & 636 & 67,4 & 943 \\
\hline SN & $1990-2014$ & 5 & 444 & 43,2 & 583 & 56,8 & 1.027 \\
\hline ST & $1990-2011$ & 5 & 417 & 46,3 & 484 & 53,7 & 901 \\
\hline SH & $1950-2009$ & 15 & 657 & 34,7 & 1.238 & 65,3 & 1.895 \\
\hline \multirow[t]{2}{*}{ TH } & $1990-2014$ & 5 & 421 & 39,7 & 640 & 60,3 & 1.061 \\
\hline & & 102 & 6.425 & 39,1 & 9.988 & 60,9 & 16.413 \\
\hline
\end{tabular}

a) Einschl. Volksinitiativen/Volksbegehren; in Bayern einschl. Entwürfe aus dem Senat.

Quelle: Eigene Berechnungen; Beiträge in Mielke/Reutter 2012; Homepages der Landesparlamente.

put beschrieben, also dargelegt, wer Gesetzentwürfe in den Landtag einbringt und ob sich unterhalb genereller Entwicklungen politikfeldspezifische Differenzierungen bemerkbar machen. (b) Sodann wird die "success rate of legislative initiatives proposed by members of the legislature" (Arter 2006b, S. 25I) untersucht. (c) Schließlich wird am Beispiel der Verfassungspolitik dem „Withinput" nachgegangen (Arter 2006b, 2006a; Beyme 1999). ${ }^{3}$

(a) Input: Bekanntermaßen beginnt Policy Making nicht mit der formalen Einbringung von Gesetzen in ein Parlament. Soweit die zielorientierte Gestaltung von Gesellschaft aber mit dem Mittel eines Gesetzes erfolgt, sind Parlamente zwangsläufig zu beteiligen. Ebenso zutreffend ist, dass eine formelle Beteiligung noch nichts aussagt über das "materielle Substrat" von Gesetzgebung, nämlich die inhaltlichen Weichenstellungen (Papier 2013, 83). Gleichwohl lässt sich prüfen, ob und inwieweit der Gesetzgebungsprozess in der Formulierungsphase durch Parlamente beeinflusst und geprägt ist. In parlamentarischen Regierungssystemen ist dies allerdings nur schwer zu erschließen, weil hier Regierung und Regierungsmehrheit eine Handlungseinheit bilden, parlamentarisches Mitregieren mithin häufig informell erfolgt und sich nicht in Parlamentaria oder

3 Die Daten und Informationen zu verfassungsändernden Gesetzgebungsverfahren beruhen auf einem von der DFG finanzierten Forschungsprojekt und sind zu finden in: Reutter 2014, 2015a, 2015b, 20I6a, 20I6c. anderen Dokumenten niederschlägt. Diese Kooperation von Exekutive und parlamentarischer Mehrheit ist einer empirischen Analyse nicht ohne Weiteres zugänglich. Dennoch erlauben die vorliegenden Befunde die Schlussfolgerung, dass Landesparlamente in dieser Phase umfassende Rechte haben und diese Rechte ausüben. Unterstellt wird dabei im Weiteren ein verfassungsrechtliches Verständnis der Landesparlamente. Sie werden sozusagen prozessstandschaftlich behandelt, d.h. Anträge und Aktivitäten von parlamentarischen Substrukturen werden dem Verfassungsorgan insgesamt zugerechnet.

Das Recht zur Gesetzesinitiative haben in allen Landesparlamenten die jeweilige Regierung sowie Fraktionen und das "Volk“. In fünf Ländern können auch einzelne Abgeordnete Gesetzentwürfe in den Landtag einbringen, in Brandenburg können dies darüber hinaus Präsident, Präsidium und Fachausschüsse. Diese Rechte sind, wie erwähnt, verfassungsrechtlich nicht gemäß den Prinzipien des neuen Dualismus ausgestaltet, sondern dem jeweiligen Parlament insgesamt bzw. dessen Substrukturen eingeräumt, unabhängig davon, ob diese zur parlamentarischen Mehrheit oder Minderheit gehören. Insoweit lässt sich konstatieren, dass Landesparlamente bzw. deren Teile in der Phase der Politikformulierung über ein Gestaltungs- oder Einflusspotential verfügen. Alle Landesparlamente - bzw. deren Fraktionen - nehmen dieses Recht in Anspruch. 
Tabelle 2 zeigt, dass die politische Exekutive rund 60 Prozent aller Gesetzentwürfe in die Landesparlamente einbringt. Fast 40 Prozent aller Entwürfe stammen aus den Landesparlamenten selbst, wobei rollentypische Ausprägungen relevant sind (Reutter 2008, 237). Koalitionsfraktionen werden nur selten unabhängig voneinander aktiv, während Oppositionsfraktionen eine höhere Anzahl von Gesetzentwürfen in die Landesparlamente einbringen, dies aber selten gemeinsam tun. Opposition kennt mithin keine Koalition. Folgerichtig wurden in Brandenburg von den zwischen 1990 und 20I4 von Oppositionsfraktionen eingebrachten I89 Gesetzentwürfen lediglich I6 angenommen. Der Rest wurde abgelehnt, zurückgezogen oder fiel dem Diskontinuitätsprinzip zum Opfer (Reutter 20I6b, 90-9I). Nicht selten werden allerdings Initiativen, die von der Opposition eingebracht wurden, von Mehrheitsfraktionen zwar zuerst abgelehnt, später aber in eigene Gesetzesvorhaben aufgenommen (Mintzel/Wasner 2012, I25-I27; Steinack 2007). Der wichtige Befund ist aber, dass Landesparlamente auf Policy Making Einfluss ausüben. Mehr noch: In der Verfassungspolitik, ein zentrales landespolitisches Thema, dominiert dieser Einfluss sogar. Verfassungspolitik stellt ein parlamentslastiges Politikfeld dar, in dem die überwiegende Anzahl von Gesetzesinitiativen aus den Landesparlamenten stammen. Durchschnittlich wurden in acht untersuchten Landesparlamenten gerade einmal 6,6 Prozent aller Gesetzentwürfe von Landesregierungen initiiert (Tabelle 3). (b) Der beschriebene landesparlamentarische Input ist ein erster Indikator, um die Performanz von Landesparlamenten und deren Einfluss auf die Staatsleitung zu umschreiben. Allerdings kommt es nicht nur darauf an, ob Gesetzentwürfe aus der Mitte des Parlamentes oder von der Regierung stammen, sondern auch auf die Erfolgschancen solcher Initiativen. Für Mezey zielen "private member bills" keineswegs auf Policy Making, da auch die Initiatoren solcher Entwürfe nur in den seltensten Fällen mit einem Erfolg rechnen (Mezey 1979, 88f.). Entscheidend ist daher die Erfolgsrate von Initiativen (Tabelle 4). Es versteht sich, dass es hier im Vergleich und im Zeitablauf - z.B. abhängig von der Parteienkonstellation - beträchtliche Änderungen und Unterschiede geben kann, zumal in den formalen Prozessen die parlamentarische Wirklichkeit sich keineswegs auf einer eins-zu-eins-Basis abbildet. So sind Regierungsentwürfe in der Regel mit den Mehrheitsfraktionen abgestimmt. Erik Damgaard und Henrik Jensen postulieren daher für Dänemark, dass die Regierung „im Schatten des Folketing“, des dänischen Parlamentes, operiere (Damgaard/Jensen 2006, 438). Das gilt auch für die deutschen Landesparlamente und zwar in doppelter Hinsicht: Die Parlamente operieren im Schatten der Landesregierungen und die Landesregierungen im Schatten der Mehrheitsfraktionen, also der Parlamente. Beide, Landesregierungen und Landesparlamente, sind funktional voneinander abhängig, bleiben aber eigenständige Verfassungsorgane.

Tabelle 3: Verfassungspolitik: Gesetzentwürfe in ausgewählten Landesparlamenten

\begin{tabular}{ccccccc}
\hline & & \multicolumn{5}{c}{ Anzahl der verfassungsändernden Gesetzentwürfe } \\
\cline { 3 - 6 } & Zeitraum & \multicolumn{2}{c}{ Aus Landtaga) } & Von Landesregierung & Gesamt \\
\hline \multirow{2}{*}{ BW } & Abs. & In \% & Abs. & In \% & Abs. \\
BAY & $1952-2011$ & 62 & 89,9 & 7 & 10,1 & 69 \\
BB & $1946-2013$ & 88 & 97,8 & 2 & 2,2 & 90 \\
MV & $1992-2014$ & 18 & 85,7 & 3 & 14,9 & 21 \\
NI & $1995-2008$ & 11 & 100,0 & 0 & 0,0 & 11 \\
NRW & $1992-2013$ & 22 & 100,0 & 0 & 0,0 & 22 \\
SN & $1950-2017$ & 56 & 86,2 & 9 & 13,8 & 65 \\
ST & $1995-2012$ & 30 & 100,0 & 0 & 0,0 & 30 \\
TH & $1992-2016$ & 5 & 100,0 & 0 & 0,0 & 5 \\
& $1990-2009$ & 31 & 93,9 & 2 & 6,1 & 33 \\
\hline
\end{tabular}

a) Einschl. Volksbegehren und Volksinitiativen. 
Tabelle 4: Anteil der verabschiedeten Gesetze an den eingebrachten Gesetzen nach Initiatoren in ausgewählten Landesparlamenten ( in \%)

\begin{tabular}{|c|c|c|c|c|c|c|c|}
\hline & \multirow[b]{2}{*}{ Zeitraum } & \multirow{2}{*}{$\begin{array}{c}\text { Anzahl } \\
\text { WP }\end{array}$} & \multirow{2}{*}{$\begin{array}{l}\text { Erfolgsquote } \mathrm{I}^{\mathrm{a})} \\
\text { alle Gesetze }\end{array}$} & \multicolumn{2}{|c|}{ Landesregierung } & \multicolumn{2}{|c|}{ Landtag } \\
\hline & & & & $\begin{array}{l}\text { Erfolgsquote } \\
\qquad\left.\right|^{\text {a) }}\end{array}$ & $\begin{array}{c}\text { Erfolgsquote } \\
\qquad \|^{\text {b) }}\end{array}$ & $\begin{array}{l}\text { Erfolgsquote } \\
\qquad\left.\right|^{\text {a) }}\end{array}$ & $\begin{array}{c}\text { Erfolgsquote } \\
\qquad \|^{\text {b) }}\end{array}$ \\
\hline \multicolumn{8}{|c|}{ Alle Gesetze } \\
\hline BB & 1990-2009 & 4 & 80,2 & 98,8 & 87,3 & 34,9 & 12,7 \\
\hline HES & 1995-2008 & 3 & 73,5 & 98,7 & 79,1 & 37,4 & 20,9 \\
\hline $\mathrm{NI}$ & 1993-2013 & 5 & 78,9 & 98,6 & 69,6 & 54,1 & 30,4 \\
\hline NRW & 1995-2017 & 5 & 86,0 & 99,7 & 82,2 & 52,7 & 17,8 \\
\hline SLD & 1970-2009 & 8 & 82,1 & 95,3 & 78,3 & 54,7 & 21,7 \\
\hline $\mathrm{SH}$ & $1992-2017$ & 6 & 71,5 & 96,7 & 72,7 & 42,7 & 27,2 \\
\hline \multicolumn{8}{|c|}{ Verfassungsändernde Gesetze } \\
\hline BW & 1952-2011 & 14 & 40,6 & 100,0 & 25,0 & 33,9 & 75,0 \\
\hline BAY & $1946-2013$ & 15 & 15,6 & 100,0 & 14,3 & 13,8 & 85,7 \\
\hline BB & 1990-2014 & 5 & 38,1 & 100,0 & 37,5 & 29,4 & 62,5 \\
\hline $\mathrm{NI}$ & 1993-2013 & 5 & 22,7 & - & - & 22,7 & 22,7 \\
\hline NRW & $1950-2017$ & 15 & 33,8 & 13,8 & 66,7 & 86,2 & 28,6 \\
\hline SN & $1990-2013$ & 5 & 3,4 & - & - & 3,4 & 100,0 \\
\hline SH & 1992-2017 & 6 & 32,7 & - & - & 34,6 & 100,0 \\
\hline
\end{tabular}

a) Erfolgsquote: prozentualer Anteil der verabschiedeten Gesetze an den von den jeweiligen Initiatoren eingebrachten Entwürfen

b) Anteil der verabschiedeten Gesetze an allen verabschiedeten Gesetzen

Quelle: Eigene Erhebungen; Parlamentsdokumentationen.

Unter Berücksichtigung dieser methodischen Einschränkungen zeigt sich bei den Landesparlamenten ein deutliches Übergewicht der Landesregierungen, wenn die Erfolgsquoten herangezogen werden. Bestimmt man die Erfolgsquote als Anteil der verabschiedeten an den eingebrachten Gesetzentwürfen der jeweiligen Initiatoren (Erfolgsquote I), zeigen sich klare Profile. Nur wenige Entwürfe der Exekutive scheitern an einer fehlenden parlamentarischen Unterstützung. Wie Tabelle 4 verdeutlicht, waren die Landesregierungen mit ihren Entwürfen fast ausnahmslos erfolgreich. Hinzu kommt, dass die Entwürfe der Exekutive auch stets den Löwenanteil aller verabschiedeten Gesetze ausmachen (Erfolgsquote II). Durchschnittlich gingen drei von vier Gesetzen, die ein Landesparlament verabschiedete, auf einen Entwurf einer Landesregierung zurück. Gleichzeitig ist zu betonen, dass ein beachtlicher Anteil der Entwürfe, die aus der Mitte der Parlamente stammten, verabschiedet wurden. In Niedersachsen, NordrheinWestfalen und dem Saarland wurde sogar mehr als die
Hälfte der aus den Landtagen stammenden Entwürfe angenommen. Hinzu kommt, dass in einzelnen Landesparlamenten mehr als jedes fünfte verabschiedete Gesetz auf einen Entwurf zurückging, der seinen Ausgangspunkt im Parlament selbst hatte.

Allerdings bestehen hier politikfeldspezifische Variationen (Tabelle 4). So fallen - im Vergleich mit der ${ }_{\text {nnor- }}$ malen" Gesetzgebung - bei Verfassungsänderungen drei Aspekte ins Auge. Zum ersten ist in diesem Politikfeld der Anteil der vergeblichen Gesetzesinitiativen signifikant höher. Jedenfalls liegt die generelle Erfolgsquote in allen Fällen weit unter 50 Prozent, in Bayern sogar bei lediglich 15,6 Prozent; in Sachsen wurde bisher gerade einmal eine Verfassungsänderung angenommen. Zum zweiten sticht hervor, dass die Landesregierungen dieses Politikfeld nur zu einem geringen Teil prägen konnten. Zwar wurde fast jeder von einer Landesregierung eingebrachte Gesetzentwurf in diesem Politikfeld angenommen; lediglich in Nordrhein-Westfalen fanden drei von neun Entwürfen einer Landesregierung keine 
Mehrheit. Gleichwohl nahm nicht einmal jeder fünfte verfassungsändernde Gesetzentwurf in der politischen Exekutive ihren formalen Ausgangspunkt. Denn für lediglich 18 von insgesamt 96 verabschiedeten Verfassungsänderungen waren Landesregierungen als Einbringer verzeichnet (= 18,8 Prozent). Dementsprechend gestalten sich zum dritten die Initiativen, die im Parlament ihren Ausgang hatten, wobei hier auch Volksbegehren und Volksinitiativen dazu gezählt werden. Zwar wird von ihnen auch nur eine Minderheit angenommen - im Durchschnitt der fünf Länder sind es gerade einmal rund 20 Prozent -, doch prägen sie zu einem überwiegenden Anteil das Politikfeld.

(c) Parlamente können nicht nur in der Entwurfsphase und in der Beschlussfassung Einfluss nehmen auf die Gesetzgebung. Vielmehr zeigt gerade die vergleichende Forschung, dass der "Within-put" (Arter 2006b) Voraussetzung für einflussmächtige Parlamente ist. Insbesondere in dem Recht, Gesetzentwürfe in Ausschüssen beraten und Änderungen vorschlagen zu können, sehen viele Studien einen wichtigen Einflussfaktor von Parlamenten (Sebaldt 2009). Auch Landesparlamente versuchen, arbeits- und redeparlamentarische Funktionsprinzipien in Einklang zu bringen (Reutter 2008, I7I-I83). Während im Plenum, so die Theorie, die öffentliche Debatte und der abschließende Beschluss im Mittelpunkt stehen, sind Ausschüsse die Orte der inhaltlichen Sacharbeit. Diese parlamentarische Arbeitsteilung funktionierte auch bei verfassungsändernden Gesetzgebungsverfahren. So verarbeiteten die Landesparlamente von Bayern, Baden-Württemberg, Brandenburg, Niedersachsen und Sachsen in 45 Wahlperioden insgesamt 23I verfassungsändernde Gesetzentwürfe, von denen 47 angenommen wurden, d.h. rund jeder fünfte. Diese Entwürfe wurden in mehr als 400 Lesungen debattiert und in knapp 300 Ausschüssen diskutiert und beraten. Diese Daten zeigen, dass Parlamente an verfassungsändernden Gesetzgebungsverfahren aktiv beteiligt waren und dass sie als Mischung aus Rede- und Arbeitsparlament funktionierten, ganz abgesehen davon, dass Empfehlungen der Ausschüsse zumeist vom Plenum angenommen wurden.

\section{Landesparlamente im Bundesstaat: tentative Schlussfolgerungen}

Der Beitrag hinterfragte die verbreitete Annahme, dass die Funktionsweise des kooperativen Föderalismus in der Bundesrepublik Deutschland einen kontinuierlichen Macht- und Einflussverlust der Landesparlamente befördere, weil sich der legislative Gestaltungsraum der Bundesländer stetig verkleinert habe und subnationale Volksvertretungen am Policy-Making nicht - mehr wirkmächtig beteiligt seien. Die Diskussion über den
Zusammenhang von Föderalismus und Parlamentarismus scheint allerdings durch eine doppelte Verkürzung geprägt (Benz 2009; Reutter 2010). Zum ersten ist zu betonen, dass sich für die These eines kontinuierlichen Machtverlustes der Landesparlamente keine belastbaren Belege finden. Im Gegenteil: Kompetenzwanderungen zwischen den staatlichen Ebenen zeitigten keineswegs immer die erwartbaren Effekte, jedenfalls führten sie nicht zu einer Negativentwicklung beim landesparlamentarischen Gesetzesoutput. Dies gilt auch für ein so wichtiges Politikfeld wie die Verfassungspolitik. Plausibler als die These einer "unheiligen Allianz" zwischen Föderalismus und Parlamentarismus scheint daher vielmehr die Annahme eines komplementären Verhältnisses zwischen Bundestag und Landesparlamenten, also eine Arbeitsteilung zwischen bundes- und gliedstaatlichen Volksvertretungen (Reutter 2006).

Zum zweiten, Landesparlamente verfügen über Einfluss auf Gesetzgebung. Wie groß dieser Einfluss ist, lässt sich empirisch nicht eindeutig bestimmen. Schon deswegen ruht die Annahme, Landesparlamente hätten in dieser Hinsicht Einflussverluste erfahren, auf unsicherem Grund. Zudem hat die Analyse gezeigt, dass Landesparlamente bzw. deren Teile in allen Stadien von Gesetzgebungsverfahren aktiv mitwirken, im Bereich der Verfassungspolitik sogar dominieren. Insoweit weist die Debatte um den Einfluss von Landesparlamenten auf Policy Making und die Staatsleitung in den Bundesländern ein Desiderat auf, weil sie diesen Einfluss systematisch vernachlässigt. Das dominierende parlamentarismustheoretische Verständnis, wie es sich etwa in einschlägigen politikwissenschaftlichen Lehrbüchern findet, löst das Verfassungsorgan „Parlament" funktional auf und begreift es ausschließlich als Teil einer Systemstruktur (Schütt-Wetschky 1992). Dieser systemtheoretische Bias hat dazu beigetragen, im Sinne des "neuen Dualismus“ Rolle und Bedeutung von Mehrheits- und Minderheitsfraktionen besser zu verstehen und damit der Gewaltenverschränkung von Exekutive und Legislative Rechnung zu tragen. In dieser Perspektive ausgeblendet bleiben aber Politikfelder und parlamentarische Bereiche, die sich nicht ohne Weiteres dem Dualismus von Regierungsmehrheit und Opposition unterordnen. Die Leistungen, die Parlamente für das politische System erbringen, lassen sich mithin erschöpfend mit den Funktionsprinzipien des parlamentarischen Regierungssystems nicht erklären. Der "alte Dualismus" lebt im „neuen“ fort. Landesparlamente sind keineswegs machtlos. 


\section{Literaturverzeichnis}

Andersen, Uwe/Rainer Bovermann (2012), Der Landtag von Nordrhein-Westfalen, in: Mielke, Siegfried/Werner Reutter (Hg.), Landesparlamentarismus. Geschichte, Struktur, Funktionen, 2. Auflage, Wiesbaden: VS Verlag, 399-430.

Arnim, Hans Herbert von (2002), Vom schönen Schein der Demokratie. Politik ohne Verantwortung - am Volk vorbei, München: Droemer.

Arter, David (2006a), Conclusion. Questioning the 'Mezey Question': An Interrogatory Framework for the Comparative Study of Legislatures, in: The Journal of Legislative Studies, Vol. I2(3-4), 462-482.

Arter, David (2006b), Introduction. Comparing the Legislative Performance of Legislatures, in: The Journal of Legislative Studies, Vol. I2(3-4), 245-257.

Benz, Arthur (2009), Ein gordischer Knoten der Politikwissenschaft? Zur Vereinbarkeit von Föderalismus und Demokratie, in: Politische Vierteljahresschrift, Vol. 5O(I), 3-22.

Beyme, Klaus von (1997), Der Gesetzgeber. Der Bundestag als Entscheidungszentrum, Opladen: Westdeutscher Verlag.

Beyme, Klaus von (1999), Die parlamentarische Demokratie. Entstehung und Funktionsweise 1789-1999. 3., völlig neubearbeitete Auflage. Opladen/Wiesbaden: Westdeutscher Verlag.

Bryce, James (192I), Modern Democracies. 2 Vols. New York: Macmillan.

Carstensen, Franziska/Suzanne S. Schüttemeyer (2015), Reden und Handeln? Zur Zukunft des Landesparlamentarismus, in: Thüringer Landtag (Hg.), Ein Vierteljahrhundert parlamentarische Demokratie. Der Thüringer Landtag 1990-20I4, Weimar: Wartburg Verlag, 282-3I4.

Crouch, Colin (2008), Postdemokratie, Frankfurt a.M.: suhrkamp.

Damgaard, Erik/Henrik Jensen (2006), Assessing Strength and Weekness in Legislatures. The Case of Denmark, in: Journal of Legislative Studies, Vol. I2(3-4), 426-442.

Dose, Nicolai/Iris Reus (2016), The Effect of Reformed Legislative Competences on Länder Policy-Making: Determinants of Fragmentation and Uniformity, in: Regional and Federal Studies, Vol. 26(5), 625-644.

Eicher, Hermann (1988), Der Machtverlust der Landesparlamente. Historischer Rückblick, Bestandsaufnahme, Reformansätze, Berlin: Duncker \& Humblot.

Eisele, Hansjörg (2006), Landesparlamente - (k)ein Auslaufmodell? Eine Untersuchung zum deutschen Landesparlamentarismus am Beispiel des Landtags von Baden-Württemberg, Baden-Baden: Nomos.

Freitag, Markus/Adrian Vatter (Hg.) (2008), Die Demokratien der deutschen Bundesländer, Opladen: Verlag Barbara Budrich.
Friedrich, Manfred (1975), Landesparlamente in der Bundesrepublik Deutschland: Opladen: Westdeutscher Verlag.

Gremmer, Bernhard (1990), Wandlungen in der Gesetzgebungsfunktion des Bayerischen Landtags von 1946 bis 1986, München: Bayerischer Landtag.

Grimm, Dieter (200I), Die Verfassung und die Politik. Einsprüche in Störfällen, München: C.H. Beck.

Habermas, Jürgen (1993), Strukturwandel der Öffentlichkeit. Untersuchungen zu einer Kategorie der bürgerlichen Gesellschaft. 3. Auflage, Frankfurt a.M.: suhrkamp.

Hennis, Wilhelm (1956/1968), Parlamentarische Opposition und Industriegesellschaft. Zur Lage des parlamentarischen Regierungssystems [zuerst 1956], in: ders., Politik als praktische Wissenschaft. Aufsätze zur politischen Theorie und Regierungslehre, München: Piper, I05-125.

Hesse, Konrad (1962). Der unitarische Bundesstaat. Karlsruhe: Verlag C.F. Müller.

Kilper, Heiderose/Roland Lhotta (1996), Föderalismus in der Bundesrepublik Deutschland, Opladen: Westdeutscher Verlag.

Klatt, Hartmut (1982), Parlamentarisches System und bundesstaatliche Ordnung. Konkurrenzföderalismus als Alternative zum kooperativen Bundesstaat, in: Aus Politik und Zeitgeschichte, B3I vom 7. August 1982, 3-24.

Kropp, Sabine (20I0), Kooperativer Föderalismus und Politikverflechtung, Wiesbaden: Verlag für Sozialwissenschaften.

Leber, Fabian (2014), Landesgesetzgebung im neuen Bundesstaat. Handlungsmuster landespolitischer Akteure nach der Föderalismusreform 2006, BadenBaden: Nomos.

Lehmbruch, Gerhard (2000), Parteienwettbewerb im Bundesstaat. Regelsysteme und Spannungslagen im politischen System der Bundesrepublik Deutschland. 3. Auflage, Wiesbaden: Westdeutscher Verlag.

Linck, Joachim (2004), Haben die deutschen Landesparlamente noch eine Zukunft?, in: Zeitschrift für Politikwissenschaft, Vol. I4(4), I215-I234.

Loewenberg, Gerhard/Samuel Charles Patterson (1979), Comparing Legislatures, Boston usw.: Little Brown and Company

Marschall, Stefan (2005), Parlamentarismus. Eine Einführung, Baden-Baden: Nomos.

Mende, Susann (2010), Kompetenzverlust der Landesparlamente im Bereich der Gesetzgebung. Eine empirische Analyse am Beispiel des Sächsischen Landtages, Baden-Baden: Nomos.

Mezey, Michael L. (1979), Comparative Legislatures, Durham: Duke University Press.

Mielke, Siegfried/Werner Reutter (Hg.), Landesparlamentarismus. Geschichte, Struktur, Funktionen, 2. Auflage, Wiesbaden: VS Verlag. 
Mintzel, Alf/Barbara Wasner (2012), Landesparlamentarismus in Bayern, in: Mielke, Siegfried /Werner Reutter (Hg.), Landesparlamentarismus. Geschichte, Struktur, Funktionen, 2. Auflage, Wiesbaden: VS Verlag, IO5-I42.

Müller, Reinhard (20II), „Der Bundestag. Kein Abnickverein." in: Frankfurter Allgemeine Zeitung vom I8. März 20II. Internet: http://www.faz.net/aktuell/politik/ inland/der-bundestag-kein-abnickverein-I604248. html (Zugriff: 20. Oktober 2016)

Norton, Philip (1990), Parliaments. A Framework for Analysis, in: ders. (Hg.), Parliaments in Western Europe. London: Frank Cass, I-9.

Oberreuter, Heinrich (2012), Substanzverluste des Parlamentarismus. Veränderungen des Verhältnisses zwischen Politik und Gesellschaft, in: Aus Politik und Zeitgeschichte, Nr. 38-39/2012, S. 25-3I.

Oberreuter, Heinrich (2013), Marginalisierung der Parlamente?, in: ders. (Hg.), Macht und Ohnmacht der Parlamente, Baden-Baden: Nomos, 2I-32.

Papier, Hans-Jürgen (20I3), Entparlamentarisierung und Selbstverantwortung der Parlamente. Ein Plädoyer für die Wahrung des demokratisch-parlamentarischen Systems, in: Oberreuter, Heinrich (Hg.), Macht und Ohnmacht der Parlamente, Baden-Baden: Nomos, 79-94.

Reutter, Werner (2006), The Transfer of Power Hypothesis and the German Länder. In Need of Modification, in: Publius: The Journal of Federalism, Vol. 36(2), 277-30I.

Reutter, Werner(2008), Föderalismus, Parlamentarismus und Demokratie. Landesparlamente im Bundesstaat, Opladen: Verlag Barbara Budrich.

Reutter, Werner (2010), Demokratie im Bundesstaat. Zum Verhältnis zweier verfassungsrechtlicher Strukturprinzipien, in: Zeitschrift für Politikwissenschaft, Jg. 2O(2), I23-I 48.

Reutter, Werner(20I4), Sächsische Verfassungspolitik, in: Europäisches Zentrum für Föderalismus-Forschung Tübingen (EZFF) (Hg.), Jahrbuch des Föderalismus 20I4. Föderalismus, Subsidiarität und Regionen in Europa, Baden-Baden: Nomos, 255-268.

Reutter, Werner (2015a), Bayerische Verfassungspolitik, in: Europäisches Zentrum für Föderalismus-Forschung Tübingen (EZFF) (Hg.), Jahrbuch des Föderalismus 20I5. Föderalismus, Subsidiarität und Regionen in Europa, Baden-Baden: Nomos, 215-227.

Reutter, Werner (2015b), Verfassungsgesetzgebung in Brandenburg. in: Zeitschrift für Parlamentsfragen, Jg. 46(I), II6-I35.

Reutter, Werner (2016a), Constitutional Politics in East Germany and the Grand Coalition State, in: Perspectives on Federalism, Vol. 8(3), 23-44.

Reutter, Werner (2016b), Der Landtag Brandenburg Abgeordnete, Struktur und Funktionen, in: Lorenz, Astrid /Andreas Anter/Werner Reutter, Politik und
Regieren in Brandenburg, Wiesbaden: Springer VS, 73-IO4.

Reutter, Werner (2016c), Verfassungspolitik in BadenWürttemberg: Ergebnis konsensdemokratischer Zwänge oder „normale Politik mit anderen Mitteln“?, in: Zeitschrift für Politikwissenschaft, Jg. 26(2), I3I-I52.

Russell, Meg/Daniel Gover/Kristina Wollter (2015), Does the Executive Dominate the Westminster Legislative Process? Six Reasons for Doubt, in: Parliamentary Affairs. Online veröffentlicht am 2I. Mai 2015, DOI: IO.IO93/pa/gsvoi6 (Zugriff: I5. Dezember 2016).

Scharpf, Fritz W./Bernd Reissert/Fritz Schnabel (1976), Politikverflechtung. Theorie und Empirie des kooperativen Föderalismus in der Bundesrepublik, Kronberg/ Ts: Athenäum.

Schmitt, Carl (1923/1988), Die geistesgeschichtliche Lage des heutigen Parlamentarismus. 8. Auflage. Berlin: Duncker \& Humblot.

Schütt-Wetschky, Eberhard (1992), Haben wir eine akzeptable Parlamentarismustheorie?, in: Hartmann, Jürgen/Uwe Thaysen (Hg.), Pluralismus und Parlamentarismus in Theorie und Praxis. Winfried Steffani zum 65. Geburtstag, Wiesbaden: Westdeutscher Verlag, 9I-II2.

Sebaldt, Martin (2009), Die Macht der Parlamente. Funktionen und Leistungsprofile nationaler Volksvertretungen in den alten Demokratien der Welt. Wiesbaden: VS.

Steinack, Katrin (2007), Opposition im Bayerischen Landtag 1994-I998. Ebenen und Strategien politischer Einflussnahme in einem hegemonialen System. Frankfurt a.M.: Peter Lang.

Thaysen, Uwe (2002), Parlamentarismus vor dem Hintergrund der europäischen Integration. Die deutschen Landesparlamente: „Hauptverlierer“ ohne Alternative, in: Politische Bildung, Jg. 35(4), 82-93.

Thaysen, Uwe (2005), Landesparlamentarismus zwischen deutschem Verbundföderalismus und europäischem Staatenverbund: Lage und Leistung 1990-2005, in: Thüringer Landtag (Hg.), Der Thüringer Landtag und seine Abgeordneten 1990-2005. Studien zu I5 Jahren Landesparlamentarismus, Weimar usw.: hain Verlag, I9-68.

\section{Autor}

Werner Reutter, Dr. phil., ist Privatdozent an der Humboldt-Universität zu Berlin und wissenschaftlicher Mitarbeiter an der Universität Leipzig. 